\title{
Diagnostic Value of Homocysteine, Cystatin C and Lipid Indices in Assessment of Cardiovascular Risk Status of Patients with Diabetes Type 2
}

\author{
Brown Holy, Joshua Marcella Tari \\ Dept. of Medical Laboratory Science, Rivers State University of Science and Technology, Npkolu, Port Harcourt, Nigeria \\ Email address: \\ hbinternationa12002@yahoo.com (B. Holy),brown.holy01@ust.edu.ng (B. Holy)
}

\section{To cite this article:}

Brown Holy, Joshua Marcella Tari. Diagnostic Value of Homocysteine, Cystatin C and Lipid Indices in Assessment of Cardiovascular Risk Status of Patients with Diabetes Type 2. European Journal of Preventive Medicine. Vol. 4, No. 3, 2016, pp. 79-84. doi: 10.11648/j.ejpm.20160403.15

Received: April 7, 2016; Accepted: April 19, 2016; Published: May 3, 2016

\begin{abstract}
Diabetes mellitus (DM) is characterized by hyperglycaemia and glycosuria due to defects in insulin secretion from the Islets of Langerhans and associated with cardiovascular disease (CVD). The imperativeness to assess effectiveness of various cardiovascular risk tools is critical with increasing rate of people with diabetes type 2. Study was aimed at the assessment and evaluation of the diagnostic relevance of homocysteine, Cystatin $\mathrm{C}$, cardiovascular risk indices in the risk profile of cardiovascular diseases in Type 2 diabetes mellitus patients. A total of 165 patients were involved in the study, of which 100 were diabetic (test) and 65 non-diabetics (control). The diabetic subjects were those whose glycated haemoglobin ( $\mathrm{HbA1C}$ ) levels were $\geq 6.5 \%$. HbA1C was estimated quantitatively by immunochemical method, Homocysteine (Hcy), Cystatin C, Lipoprotein (a) (Lp (a) were measured by Enzyme Linked Immunosorbent assay (ELISA) method; Glucose oxidase method was used for the determination of fasting plasma glucose (FBS). The results showed elevated serum homocysteine (Hcy), Lp (a) among the diabetics as compared to the non-diabetic subjects $(p<0.05)$. The diabetic subjects also depicted higher Cystatin $\mathrm{C}$ (Cys C) levels, in the diabetic type 2 patients compared to the non-diabetic patients. The lipid profile levels in the diabetic subjects showed higher mean values of total cholesterol and triglycerides, $(p<0.05)$ as compared with the non-diabetic. The percentage risk predictive values of cardiovascular disease in the diabetic sub-population with these risk indices showed Hcy with a sensitivity of $96 \%$, specificity of $96.9 \%$ and an accuracy of $96.5 \%$ as compared to Cys $\mathrm{C}$ with a sensitivity of $95 \%$, specificity of $96.9 \%$, accuracy of $95.9 \%$ also compared with that of Lp (a) with a sensitivity of $91 \%$, specificity of $100 \%$ and an accuracy of $95.5 \%$. The level of accuracy of Hcy, Cys C and then Lp (a) amongst other indices showed that these diagnostic tools could provide better platform in cardiovascular risk profiling of T2DM patients and should be encouraged.
\end{abstract}

Keywords: Diabetes, Cardiovascular, Risk, Cystatin C, Homocysteine, Lipids

\section{Introduction}

The prevalence of cardiovascular disease worldwide can be ascribed to the global epidemic of diabetes type 2 [1]. However, history of chronic kidney disease, hypercholesterolemia and cardiovascular disease are life threatening to the patients with type 2 diabetes mellitus [2]. According to the National Cholesterol Education Program (NCEP/Adult panel Treatment, ATP Panel-III), the use of some certain traditional predictive measures such as gender, cholesterol, diabetes mellitus, hypertension, smoking and family history of premature Cardiovascular disease (CVD) are quite robust and leave room for improvement with more aggressive novel indices, these indices include homocysteine, cystatin C, lipoprotein (a) amongst others. The NCEP/ATPIII recommends the use of these indices as they can give long term predictive models to improve CVD risk prediction. [3] proposed the notion of hyperhomocysteinemia as an independent cardiovascular risk factor. Since then, evidence continuously emerged that an elevated plasma homocysteine level in the range of $15-25 \mu \mathrm{mol} / \mathrm{L}$ correlated with Coronary Heart Disease (CHD), stroke, peripheral artery stenosis and 
venous thrombosis [4].

Homocysteine is an amino acid, amino acid are the building blocks of protein. It is not possible to get homocysteine from the diet [5]. If homocysteine cannot be converted into cysteine or returned to the methionine form, levels of homocysteine in the body increase and elevated homocysteine levels have been associated with heart attack, stroke, blood clot formation and perhaps the development of Alzheimers disease [6]. Elevated homocysteine levels are associated with increased risk factors such as smoking, high blood pressure, high cholesterol or diabetes; and as such patients need to be diagnosed for homocysteine levels in the blood. The conventional lipid profile is a panel of blood tests that serves as an initial broad medical screening tool for abnormalities in lipids such as cholesterol and triglycerides may not provide definitive result upon which treatment can be based. This measure is the conventional test done in the laboratory in assaying for patients with cardiovascular disease [3].

According to the World Health Organization (WHO), the prevalence of diabetes for all age groups worldwide was estimated to be $2.8 \%$ in 2000 and $4.4 \%$ in 2030 [7], while the estimation of the prevalence of earlier stages of Chronic Kidney Disease (CKD) in the US population and occurrence in trends overtime and was focused to disease management and prevention planning of which an increased intervention was given to the prevalence of diabetes and obesity [8]. To prevent this increase, it was necessary to screen for the early detection of CKD to achieve early diagnosis. In diabetic patients, the early detection of diabetic nephropathy has focused on the measurement of serum level of Cystatin C. Cystatin C, a cysteine protease inhibitor, freely filtered by the renal glomeruli metabolized by the proximal tubule was identified as a promising marker of renal failure [9]. Cystatin $\mathrm{C}$ is produced at a constant rate by nucleated cells and released into bloodstream with a half -life of 2 hour. It's concentration is almost totally dependent on the Glomerular Filtration Rate (GFR) [10].

Therefore, estimation of the GFR is essential for the evaluation of patients with chronic kidney disease and its usefulness a tool to screen in high-risk groups as persons with diabetes mellitus [11]. Furthermore, estimation of GFR, derived from modification of Diet in Renal Disease, MDRD formula was recommended in animal evaluation of all patients with type 2 diabetes mellitus [12]. Recently, serum Cystatin C, low molecular- weight protease inhibitor, freely filtered across the glomerular membrane and then reabsorbed and metabolized in the proximal tubule was proposed as a new endogenous marker of GFR [13]. However reports have suggested that serum Cystatin concentration is a better indicator of GFR than serum creatinine concentration in patients with diabetes [14].

For an effective regiment to be put into place, a 2012 study of multiple data base including 50,000 people with coronary heart disease called the potential for a cause and effective relationship between homocysteine levels and heart disease was put into question [15]. The relationship between homocysteine levels and heart stroke as well as Cystatin $\mathrm{C}$ which is not only a sensitive biomarker of renal dysfunction but levels predicts incidence of diabetics independently of glycaemic and cardiovascular events [16]. Therefore the aim of the research is to determine the relevance of Homocysteine and Cystatin $\mathrm{C}$ in the assessment of the cardiovascular risk profile of patients with type 2 diabetes mellitus.

\section{Materials and Method}

\subsection{Subjects Characterization and Design}

One hundred and Sixty-five $(n=165)$ subjects of both male and female were recruited in this study, out of which one hundred (Females=56 and Males=44) were diabetes type 2 patients and the remaining sixty-five non- diabetic patients (Females=37 and Males=28) were the control group. The patients were all screened to ascertain their glycaemic control and this was achieved through the use of the multi test $\mathrm{A} 1 \mathrm{C}$ Now kit; the test groups which were the type 2 diabetes mellitus patients showed levels of glycated hemoglobin $\mathrm{HbA1C}, \geq 6.5 \%$ while the non-diabetic control group showed glycated heamoglobin level below $6.5 \%$.

\subsection{Sample Collection and Preparation}

Blood samples were collected from the subjects by venous puncture procedure. Suitable and prominent vein was selected and collection site was sterilized with cotton wool soaked with $70 \%$ alcohol (spirit). Five milliliters $(5 \mathrm{ml})$ of blood was collected using a $5 \mathrm{ml}$ syringe out from which 1 milliliter of blood was put into lithium heparin bottle to ascertain for glycated haemoglobin, $\mathrm{HbA1C}, 1$ milliliter for Fasting Blood Sugar, FBS in a fluoride oxalate bottle and then spun to ascertain for fasting blood sugar using the glucose oxidase method. The remaining 3 milliliter of blood was put into a plain bottle and then spun to ascertain for the other test (Homocysteine, Cystatin C, Lipoprotein (a) and Lipid profile). The sample were allowed to clot for two hours at room temperature before centrifuging for 20 minutes at approximately $3000 \mathrm{r}$. p. $\mathrm{m}$ and samples were stored in aliquot and refrigerated to remain frozen.

\section{Results}

\subsection{Cardiovascular Risk Markers/Indices in the Diabetics as Compared with That of the Non Diabetic Subjects}

The details cardiovascular risk markers/indices: Homocysteine, Cystatin C, Cholesterol/HDL Ratio and TETRAD as risk markers of diabetics and non diabetic subjects are presented in Table 1 and Figure 1 to 5 . There were elevated values of Homocysteine, and cystatin $\mathrm{C}$ in the diabetic subjects as compared to the non diabetic control subjects, $(\mathrm{p}<0.05)$. 

Assessment of Cardiovascular Risk Status of Patients with Diabetes Type 2

Table 1. Risk markers /Indices mean values of diabetic values compared with non diabetic control.

\begin{tabular}{llll}
\hline Risks Markers/Indices & Control & Diabetic Subjects & p-values \\
\hline Hcy $(\mathrm{umol} / \mathrm{L})$ & $7.61 \pm 4.18$ & $10.50 \pm 3.50$ \\
CYSC $(\mathrm{mg} / \mathrm{L})$ & $0.59 \pm 0.05$ & 0.0001 \\
CHOL $/ \mathrm{HDL}$ RATIO & $4.78 \pm 1.48$ & 0.22 & 0.0001 \\
TETRAD $(\mathrm{mmol} / \mathrm{L})$ & $474.72 \pm 300.06$ & 0.0001 & 3.86 \\
\hline
\end{tabular}

(Hcy=Homocysteine, $\mathrm{CYSC}=\mathrm{Cystatin} \mathrm{C}, \mathrm{CHOL} / \mathrm{HDL}$ RATIO=Cholesterol/High Density Lipoprotein Ratio)

$$
\text { P value } 0.0001
$$

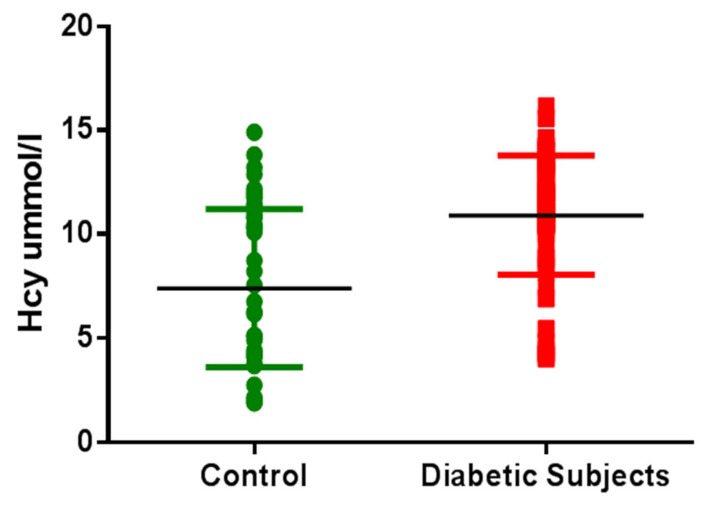

- Control

- Diabetic Subjects

Figure 1. Homocysteine mean values of diabetic subjects compared with control.

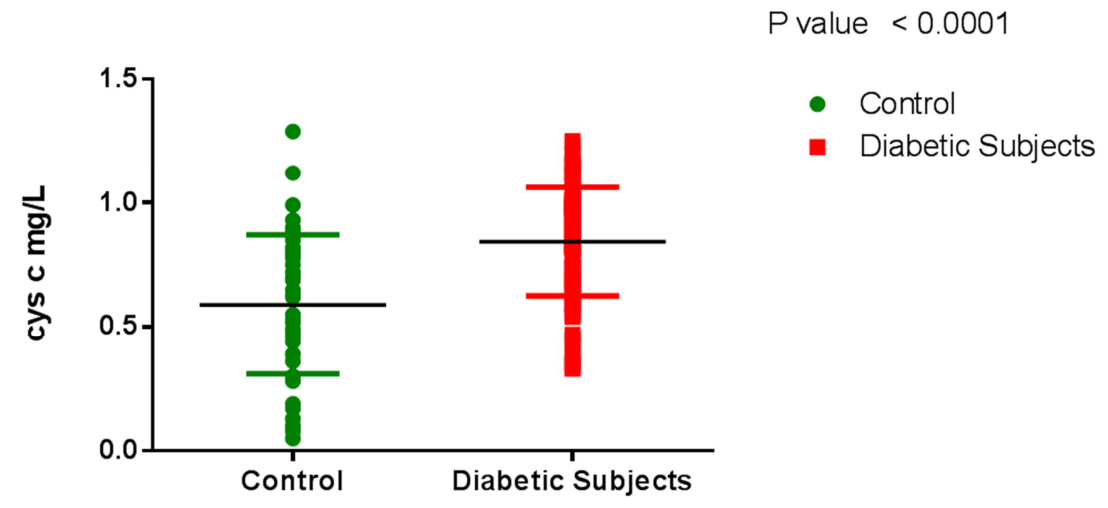

Figure 2. Aligned Dot Plot of Cystatin C values of diabetic subjects compared with non diabetic control.

$$
P \text { value }<0.0001
$$

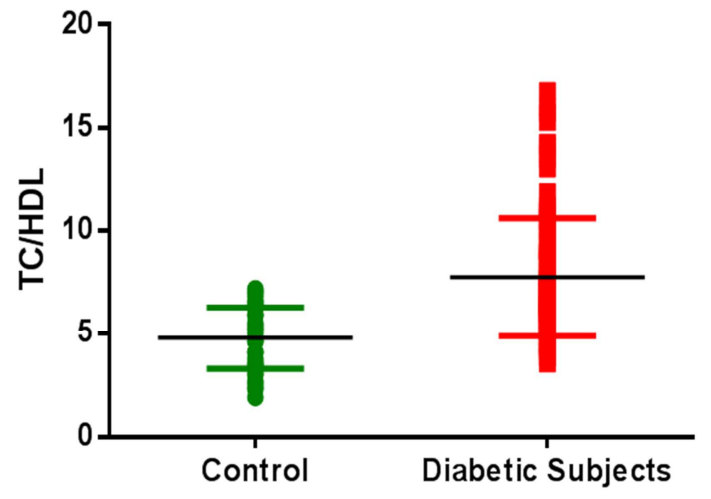

- Control

- Diabetic Subjects

Figure 3. Aligned Dot Plot of Total Cholesterol/High Density Lipoprotein Ratio of diabetic subjects compared with the control. 


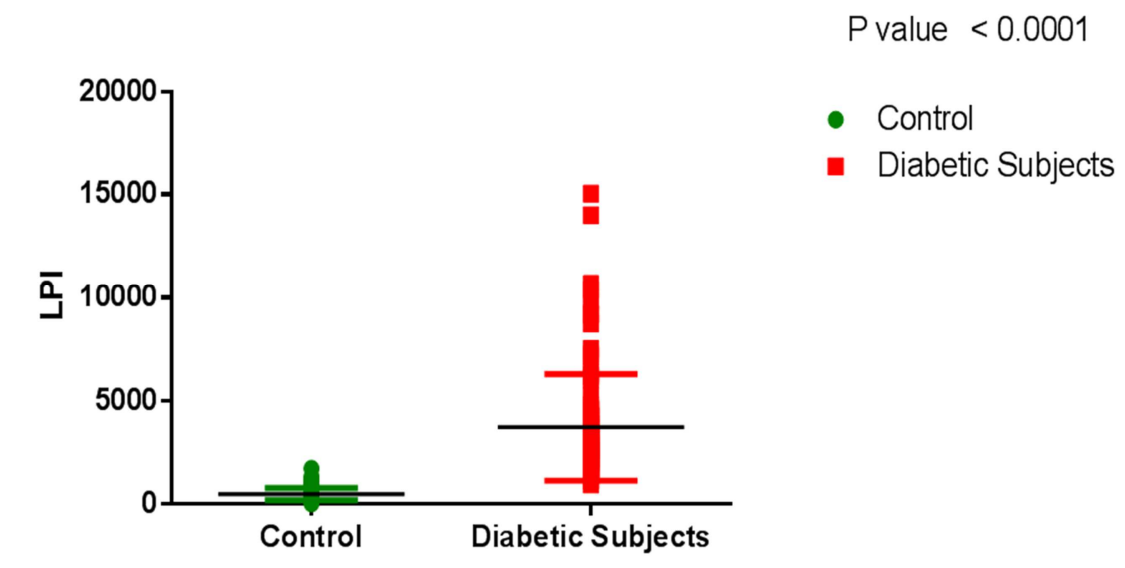

Figure 4. Aligned Dot Plot of Lipoprotein (a) values of diabetic subjects as compared with the control.

$P$ value $<0.0001$

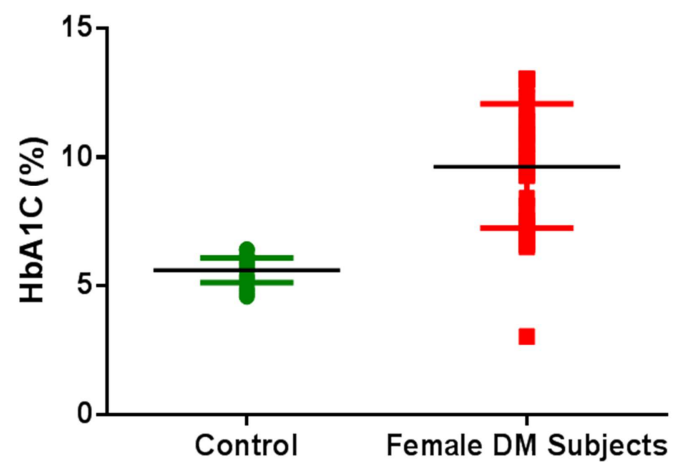

- Control

- Female DM Subjects

Figure 5. Aligned Dot Plot of Glycated Haemoglobin values of female diabetic subjects compared with the non diabetic control.

Table 2. Risk Indices of HCY, CYSC, Lp (a), TCHOL/HDL and TETRAD showing Sensitivity\%, Specificity\%, Relative Risk, Positive predictive value, Negative Predictive value and Accuracy of diabetic population.

\begin{tabular}{llllll}
\hline RISK INDICES \% & SENTIVITY \% & SPECIFICITY \% & $\begin{array}{l}\text { POSITIVE PREDICTIVE } \\
\text { VALUE \% }\end{array}$ & $\begin{array}{l}\text { NEGATIVE PREDICTIVE } \\
\text { VALUE \% }\end{array}$ & ACCURACY \% \\
\hline $\mathrm{HCY}>13$ & 96 & 96.9 & 98 & 94 & 96.5 \\
$\mathrm{CYS} \mathrm{C}>1.2$ & 95 & 96.9 & 97.9 & 92.7 & 95.9 \\
$\mathrm{LPa}>30$ & 91 & 100 & 100 & 87.8 & 95.5 \\
$\mathrm{TC} / \mathrm{HDL}>4.0$ & 97 & 35.4 & 69.7 & 88.5 & 66.2 \\
TETRAD $>20,000$ & 76 & 100 & 100 & 73 & 88 \\
\hline
\end{tabular}

( $\mathrm{HCY}=$ Homocysteine, $\mathrm{CYS} \mathrm{C}=$ Cystatin $\mathrm{C}, \mathrm{LPa}=$ Lipoprotein (a), TC/HDL=Total Cholesterol Ratio)

\subsection{Predictive Risk Indices (\%) Indicating the Sensitivity, Specificity, Relative Risk, Positive Predictive Value, Negative Predictive Value and Accuracy}

From Table 2, the percentage predictive risk of the cardiovascular indices in the diabetic sub population for Homocysteine (HCY) was sensitivity 96\%, specificity $96.9 \%$. Cystatin C with a sensitivity of $95 \%$, specificity of $96.9 \%$ and a lesser accuracy of $95.9 \%$, while Lipoprotein (a) showed a sensitivity of $91 \%$, specificity of $100 \%$ and a more lesser accuracy of $95.5 \%$. This assessment however showed Homocysteine as a more accurate biomarker/index in the profiling of cardiovascular risk in patients with type 2 diabetes as compared to Cystatin $\mathrm{C}$ and then lipoprotein (a).

\section{Discussion}

Growing interest is being focused on the association of homocysteinemia with diabetes type 2 complications and Cardiovascular complications present a leading cause of mortality in patients with diabetes mellitus type 2 [17].

In this present study, the results showed significantly elevated serum homocysteine levels among diabetics compared to non diabetic subjects $(\mathrm{p}<0.05)$, this is in agreement with the work of [18] who determined biochemical parameters and anthropometric indices among 70 diabetic subjects with results indicating significant increase in homocysteine with increase in Body Mass Index.

These data demonstrate that high serum homocysteine 
concentrations are associated with an increased risk of cardiovascular diseases independent of traditional risk factors in patients with renal disease. This study was same as that demonstrated by [19] who demonstrated substantial homocysteine uptake and metabolism by the rat kidney in vivo with only trial urinary excretion. Extrapolating these findings to humans suggest that two- thirds of plasma homocysteine elimination may be related to renal metabolism.

The diabetic subject also depicted significantly high cystatin C levels compared to non diabetic subjects. This is in consonance with the work done by [20] that showed that cystatin C levels, independent of renal function was associated with insulin resistance and inflammation which may explain the association of cystatin $\mathrm{C}$ and cardiovascular disease in Type 2 diabetes mellitus patients.

Moreover, the concentration of lipoprotein (a), Lp (a) was higher in that of the diabetics than that of the non diabetics, this is suggestive that such patients with high concentrations of lipoprotein (a) are at higher risk of developing future Coronary Heart Disease (CVD) [21]. However when Lp (a) concentrations were compared with that of male and female diabetic group, it was observed that concentration of Lp (a) was higher in diabetic female subjects as compared to that in male diabetics, this did not agree with that of [22] who stated that associations between elevated Lp (a) and Coronary Heart Disease are less robust in women than in men possibly because of the cardio protective and vasoprotective effects of endogenous estrogen in premenopausal women. He further stated that in women, elevated Lp (a) may function as a stronger risk factor when combined with elevated inflammatory or thrombotic markers. A number of studies have shown possible adverse effects of homocysteine on endothelium, platelets and coagulation factors. In a related study, homocysteine is ultra filtered through the glomeruli, almost completely reabsorbed in the tubules and degraded in the kidney tissue by transmethylation and transulfuration [23]. Recently serum Cystatin $\mathrm{C}$ has been implicated as a more sensitive marker for mildly impaired Glomerular Filtration Rate (GFR) and also as an independent determinant of serum homocysteine concentrations [24]. [25] suggested that the increase of serum homocysteine concentrations with advancing age-related decline in renal function.

The effect of various lipid parameters as well as lipoprotein (a) on the atherogenecity is not additive but multiplicative which is well demonstrated by the lipid tetrad index. The mean tetrad index of the patients with type 2 diabetes mellitus were significantly higher than the patients without type 2 diabetes mellitus, this was in consonance with the review done by [26] which showed that Asians had a deadly tetrad index which becomes the single predictor of cardiovascular disease in Asian region. The percentage predictor of cardiovascular disease in the diabetic sub population of the risk indices showed homocysteine with a sensitivity of $96 \%$, specificity of $96.9 \%$ and an accuracy of $96.5 \%$ as compared to cystatin C with a sensitivity of $95 \%$, specificity of $96.9 \%$, accuracy of $95.9 \%$ also compared with that of $\operatorname{Lp}$ (a) with a sensitivity of $91 \%$, specificity of $100 \%$ and an accuracy of $95.5 \%$. This however showed that homocysteine with higher percentage accuracy as compared to other indices is a better biomarker in the assessment of cardiovascular risk profile in patients with type 2 diabetes mellitus.

\section{Conclusion}

The diagnostic and predictive value of the homocysteine, cystatin $\mathrm{c}$ and lipid indices in this study has come into focus with homocysteine demonstrating highest accuracy followed by cystatin c. Lpa has the best positive predictive value hence may be a better tool for screening. Further work needed to be done to establish the reference range of the tools in the black population.

\section{References}

[1] Katarzyna, K., Artur, C., Ewa, C., and Anna, J., (2015). Homocysteine - relation to hypertension, age and smoking in patients with newly diagnosed essential hypertension. Journal of Medical Science, 2 (82), 90-95.

[2] Wang, X., Qun, X., Dermritas, H., Li, T. Mao, G. Huo, Y. Sun, N. \& Liu, L. (2007). Efficacy of folic and supplements in stroke prevention, a meta analysis. Lancet, 369, 1876-1882

[3] McCully, K. S. (2011). Vascular pathology of homocysteine implications for the pathogenesis of atherosclerosis, American Journal of Pathology, 56, 111-128

[4] Den H. M., Rosendaal F. R., Blom H. J., Gerrrits W. B, Bos, G. M (1998). Hyperhomocysteine and venous thrombosis, a meta-analysis. Journal of Thrombosis and Haemostasis 80, 874-877

[5] Ward M., McNulty H., Pentieva K., McPartlin J., Strain J. J., Weir D. G. \& Scott J. M.(2000). Fluctuations in dietary methionine intake do not alter plasma homocysteine concentration in healthy men, Journal of Nutrition, 130 (11), 2653-2657

[6] Clarke, R., Peden, J. F, Hopewell, J. C. (2013). PROCADIS consortium, Genetic variants associated with Lp (a) Lipoprotein a, levels and coronary disease. Journal of New England of Medicine, 361 (26), 2518-2528

[7] Wild, S., Roglic, G., Green, A., Sicee, R. \& King, H. (2004). Global prevalence of diabetes. Estimates for the year 2000 and projections for 2030. Journal of Diabetic Care, 27,10471053

[8] Gregg E. W., Cheng Y. J., Cadwell B. L., Imperatore G, William D. E., Flegal, K. M., Narayan \& K. M., Williamson, D. F (2005). Secular trends in cardiovascular disease risk factors according to body mass index in US adults. Journal of American Medical Association, 293, 1868-1874

[9] Yun K. J., Mi R. K., Jung E. H., Ji Y. M., Sang H. S., Sang S. K., Bo H. K., Soo H. L., Yong K. K. \& In J. K. (2011). Cystatin $\mathrm{C}$ as an Early Biomarker of Nephropathy in Patients with Type 2 Diabetes, Endocrinology, Nutrition \& Metabolism, Journal of Korean Medical Science, 26, 258-263 
[10] Pucci, L., Triscornia, S., Lucchesi, D. (2007). Cystatin C and estimates of renal function: searching for a better measure of kidney function in diabetic patients. Journal of Clinical Chemistry, 53, 480-488

[11] National Kidney Foundation (2002). Clinical practice guideline to define chronic kidney disease evaluation, classification and stratification. American Journal of kidney

[12] American Diabetes Association (2003). Management of dyslipidemia in adults with diabetes mellitus type 2 (position statement). Journal of Diabetes Care, S83-S86

[13] Dhia, R. B, Hellara, I., Harzallah, O., (2012): Evaluation of the renal function in type 2 diabetes, clearance calculation or cystatin c. Analyses de Biologic Clinique 70, (3), 287-294

[14] Hojs, R., Bevc, R., Ekart, M., Gorenjak, Puklaverc, L. (2008). Serum cystatin $\mathrm{C}$ - based equation compared to serum creatinine-based equations for estimation of glomerular filtration rate in patients with chronic kidney disease. Journal of Clinical Nephrology, 70, 10-17

[15] Clark, R., Halsey, J., Lewington, S., Lonn, E., Armitage, J., Manson, J. E., Bonna, K. H., Spence, J. D., (2012). Effect of lowering homocysteine levels with B vitamins on cardiovascular disease, cancer and cause specific mortality, and meta-analysis of 8 randomized trials involving 37, 485 individuals. Arch International Medicine 170, 1672-1631

[16] Wang, S. R., Ynz, H. C., Messias, A. C. \& Floegel, A. H., (2012). Novel biomarkers for pre-diabetics identified by metabolomics. Molecular System Biology,. 8, 615

[17] Mazza A, Bossone E, Mazza F, Distante A. (2005). Reduced serum homocysteine levels in type 2 diabetes. Nutr Metabolism Cardiovasc Dis; 15: 118-24

[18] Ebesunun, M. O., \& Obajobi, E. O. (2012). Elevated plasma homocysteine in type 2 diabetes mellitus: a risk factor for cardiovascular diseases. The Pan African Medical Journal, 12, 48 .

[19] Bostom A., Brosnan J. T, Hall, B, Nadeau M. R and Selhub, J. (1995): Net uptake of plasma homocysteine by the rat kidney in vivo, Atherosclerosis, 116, 59-62

[20] Alaaeldin, M. B., Ayman, A. S. F., Nada, A., Puja, M. T., Elamin, A., \& Fauzia, R. (2015). Cystatin C and Its Role in Patients with Type 1 and Type 2 Diabetes Mellitus, Advances in Endocrinology, 1-6

[21] Robinson, K., Clark R., Naughton, E., Cahalane, S., (1995): Hyperhomocysteinemia an independent risk factor for vascular disease. New England Journal of Medicine 324, $1149-1155$

[22] Shai, I., Jiang, R., Manson J. E, Stampfer, M. J., Willett, W. C., Colditz, G. A, Hu, F. B (2004): Ethnicity, Obesity, and Risk of Type 2 Diabetes in Women, A 20-year follow-up study, Circulation, 2824-2830

[23] Dudman, N. P. B (1999): An alternative view of homocysteine, Lancet, 354, 2072-2074

[24] Randers, E., Erlandsen, EJ, (1999): Serum cystatin C as an endogenous marker of the renal function: a review, Clinical Chemistry and Laboratory Medicine 7, 389-395

[25] Norlund L., Grubb A., Fex G., Lksell H., Nilsson J. E., Schenck H., Hultberg B. (1998). The increase of plasma homocysteine with age is partly due to the deterioration of renal function as determined by plasma cystatin C. Clinical chemistry and Laboratory Medicine, 36, 175-178

[26] Yeolekar, M. E. (2010). Coronary artery disease in Asian Indians, Journal of Post Graduate Medicine 44, 26 